\title{
OPEN Development and validation of a new instrument to measure social pain
}

\begin{abstract}
Ulrich Stangier $^{1 凶}$, Johanna Schüller ${ }^{1} \&$ Elmar Brähler ${ }^{2}$
Social pain is an emotional reaction to social exclusion which has been widely investigated in experimental settings. We developed the Social Pain Questionnaire (SPQ) and examined its factor structure, reliability, and construct validity. We constructed a 46 -item pool that covered a broad range of situations related to social pain. Using three different subsamples (Online convenience sample: $n=623$, Representative sample: $n=2531$, Clinical sample of outpatients seeking psychotherapy: $n=270$ ) we reduced the item pool to 10 items for the final SPQ scale, paying particular attention to content validity and factorial structure. Convergent, divergent and discriminant validity were assessed using standardized measures of related constructs and group differences. For the final 10-item version, a good factorial structure and reliability were found. Convergent validity was supported by correlations with related instruments of interpersonal sensitivity, attachment styles, depression and social anxiety. The representative and clinical sample differed significantly in social pain. The SPO is an economic self-report measure with solid psychometric properties. Our data support the factorial, construct and convergent validity. The SPQ can be used to clarify the role of social pain in mental disorders and to incorporate interventions targeted towards social pain in psychotherapeutic settings.
\end{abstract}

Social pain has been defined as a negative emotional state triggered by the perception of being excluded or rejected from a group or a relationship to a significant person ${ }^{1}$. However, the construct has been conceptualized in different ways concerning triggers, quality of emotional states and psychological mechanisms involved ${ }^{2}$.

The feeling of "hurt" is a negative emotional state, which is different from other social emotions such as fear, shame or guilt. Due to the subjective overlap with physical pain concerning intensity and adversity, as well as similarities in the neurobiological processing ${ }^{4}$, the term "social pain" has become more common in literature. The feeling of being hurt has been conceptualized as a reaction to interpersonal rejection, associated with a relational devaluation ${ }^{5}$. In contrast, social pain has been more often used in the context of social exclusion and ostracism, to characterize the target's reaction to threats for the need for belonging ${ }^{1,4}$. Both rejection and exclusion are not restricted to specific social domains but may occur in romantic relationships, kin and family, friends, peers, or groups ${ }^{6}$.

The social-physical pain overlap theory ${ }^{4}$ suggests that both systems share common physiological mechanisms because evolutionarily, rejection and social exclusion posed similarly serious threats to survival as physical injury. However, this theory has been criticized, since data from fMRI studies show inconsistent results with regard to the activation patterns of the brain involved in social pain, and also indicate that this pattern is neither specific for social pain nor for pain ${ }^{7}$. Besides the controversy about the neurobiological basis for social pain, however, there is consensus that the cyberball paradigm which has been used in most of these studies is a reliable and valid experimental paradigm to induce social pain as a reaction to social exclusion ${ }^{8}$.

Extreme sensitivity to rejection, relational devaluation and perceived social exclusion are included in the criteria for several psychiatric diagnoses, especially social anxiety disorder (SAD), depression and borderline personality disorder (BPD) ${ }^{9}$. Furthermore, early emotional neglect, abuse, and chronic rejection during childhood are important risk factors for the later development of these disorders ${ }^{10}$. Research focusing on the relationship of social pain to psychopathology focused mainly on two methodological approaches. Using self-report measures, significantly increased fear of rejection was found in patients with depression, personality disorders, and SAD ${ }^{11}$, among the personality disorders most prominently $\mathrm{BPD}^{12}$. Interestingly, using the cyberball paradigm, a similar pattern evolved for diagnoses with high ratings of need threat ${ }^{13}$ : patients with BPD and chronic depression ${ }^{14,15}$,

${ }^{1}$ Department of Clinical Psychology and Psychotherapy, Goethe-University, Frankfurt, Germany. ${ }^{2}$ Department for Psychosomatic Medicine and Psychotherapy, University Medical Center of Johannes Gutenberg University of Mainz, Mainz, Germany. ${ }^{\circledR}$ email: stangier@psych.uni-frankfurt.de 
as well as $\mathrm{SAD}^{16}$, but not posttraumatic stress disorder ${ }^{17}$ showed significantly more fear of rejection than healthy controls.

However, methodological problems limit the interpretation of these results. In the cyberball experiment, assessment of social pain is based on the needs-threat scale ${ }^{18}$. The needs-threat model ${ }^{19}$ assumes that social exclusion threatens four fundamental needs: belonging, self-esteem, control and meaningful existence. However, construct validity has been questioned due to high intercorrelations and inconsistent correlations with other questionnaires assessing diverging need ${ }^{20}$. Furthermore, it should be noted that the need-threat scale is related to short-term emotional reactions to the experience of being excluded in the cyberball game, thus covering rather a response to a specific situation than a consistent response tendency to perceive social pain in different situations.

Among standardized self-report instruments, the Adult Rejection Sensitivity Questionnaire (ARSQ) ${ }^{21}$ refers to anxious expectations in situations, in which the rejection of a request by significant others is anticipated, but not actually experienced. The Interpersonal Sensitivity Measure (IPSM) ${ }^{22}$ focuses on excessive awareness and vigilance of the behavior and feelings of others, particularly on perceived or actual criticism or rejection. However, the theoretical basis of this measure refers to the concept of 'depression-prone' personality, but is also derived from the clinical experience of the authors. The items of the IPSM assess emotional reactions to a broad range of interpersonal situations, covering not only fear of rejection but also susceptibility to critique, self-criticism, anxiety, shyness and dependency. In addition, besides the unclear theoretical relationship to social pain, subsequent studies did not support the original dimensional structure either of the ARSQ ${ }^{23}$ or the IPSM ${ }^{24}$. Finally, the Hurt Feelings Scale by Leary and Springer ${ }^{3,25}$ is a six-item scale referring to hurt as specific emotion triggered by interpersonal rejection, developed based on qualitative interviews on emotional states following hurtful episodes. Although the Hurt Feelings Scale is more specifically related to the emotional components of social pain than the other instruments mentioned before, its psychometric properties have not been evaluated systematically. The items have been derived from content analyses of qualitative interviews referring to interpersonal triggers in daily life, but a modification referring to Hurt Feeling Proneness has not been validated to our knowledge. To conclude, there is a lack of standardized and validated instruments specifically related to the conceptual framework of perceived social pain as a response predisposition, which are appropriate also for clinical settings.

This article describes the development and validation of a new scale focusing on the assessment of social pain. We present data from three substudies:

1. Generation of an initial item pool and assessment of psychometric criteria in a self-selected online sample.

2. Two-step reduction to 10 items, using a genetic algorithm.

3. Assessment of the factorial structure, and the convergent, divergent and discriminant validity of the final scale.

\section{Results}

Substudy 1: development of an item pool. The theoretical basis for the construction of an item pool for the Social Pain Questionnaire (SPQ) was to cover different emotional and embodied cognitive responses to interpersonal situations related to social exclusion and interpersonal rejection. The item pool contained 46 statements referring to diverse everyday interpersonal situations related to rejection or exclusion with friends, romantic partners, family, acquaintances, peer groups and professional relationships ( 31 items), as well as unspecified situations with others (15 items). The responses described in the statements were either related to emotional responses to others' behaviors (15 items), embodied cognitions/interpretation of the others' behaviors (14 items), or both (17 items). Based on entries in the most popular German Thesaurus (www.duden.de), synonyms were used for social or mental pain in German language, such as "hurt" ("verletzt"), "offended" ("gekränkt"), "rejected" ("zurückgewiesen") or "feel affected" ("weh tun"). The items are evaluated on a five-point Likert scale ranging from $0=$ "Applies not at all to me" to $4=$ "Applies exactly to me" Although intensity ratings are more common in emotion research, appropriateness appears to be more suitable for the judgement of a complex interaction of situation, emotional response and interpretation of the behavior of others. To form the total score, the mean of all item scores is calculated. This preliminary item pool was presented to a self-recruited online sample for a first assessment of the factorial structure. The model fit in the complete item pool was poor $\left(\chi^{2}=5071.146\right.$, $\mathrm{p}<0.001$; CFI $=0.671$, RMSEA $=0.085$, SRMR $=0.071$, indicating levels of misspecification. Modification indices showed several error covariances, indicating problems with the unidimensionality of the instrument. In the second substudy, we, therefore, reassessed the item pool, to improve the construct definition and model fit.

Substudy 2: item reduction. In the first step, a preliminary reduction was performed to minimize redundancy and clarify construct specification within the item pool. The intermediate version was then presented to a representative and a clinical sample. Using a meta-heuristic search algorithm, the final reduction to ten items was performed to optimize model fit and discrimination between patients and healthy participants.

First item reduction. Factor loadings in the initial item pool ranged between $\lambda=0.30$ and $\lambda=0.68$. We first deleted 24 items with loadings $\leq 0.60$. We additionally identified items clusters for which modification indices indicated different foci of situation type (i.e. romantic relationships, friendships, groups, acquaintances, colleagues) and deleted four items with non-specified situation types to retain a balanced range of specific and general situations within a one-dimensional construct definition. The remaining item pool comprised 18 items which covered a broad range of situations associated with social pain, including a balanced number of specific and general interpersonal situations. Most of the items referred both to emotional responses as well as embedded cognitive aspects, six items focused on emotional responses and one on cognitive aspects (i.e. interpretation of being excluded). Factor loadings of the item pool and selected items can be found in Supplementary Table S1. 


\begin{tabular}{|l|l|r|l|l|}
\hline & Sample & N & M/Md & SD/IQR \\
\hline SPQ $(18)^{\mathrm{a}}$ & Representative & 2531 & 1.72 & 1.33 \\
\hline SPQ $(10)^{\mathrm{a}}$ & Representative & 2531 & 1.80 & 1.40 \\
\hline SPQ $(18)^{\mathrm{b}}$ & Clinical & 270 & 2.21 & 0.79 \\
\hline SPQ $(10)^{\mathrm{b}}$ & Clinical & 270 & 2.25 & 0.83 \\
\hline PHQ $^{\mathrm{a}}$ & Representative & 2519 & 1.25 & 0.75 \\
\hline GBB $^{\mathrm{a}}$ & Representative & 2529 & 1.38 & 0.88 \\
\hline BSI-somatization $^{\mathrm{a}}$ & Clinical & 265 & 0.43 & 0.86 \\
\hline BSI-interpersonal sensitivity $^{\mathrm{a}}$ & Clinical & 263 & 1.00 & 1.5 \\
\hline BSI-depression $^{\mathrm{a}}$ & Clinical & 267 & 0.83 & 1.33 \\
\hline BSI-phobix anxiety $^{\mathrm{a}}$ & Clinical & 267 & 0.40 & 0.80 \\
\hline BSI-paranoid ideation $^{\mathrm{a}}$ & Clinical & 268 & 0.60 & 1.20 \\
\hline IPSM $^{\mathrm{b}}$ & Clinical & 260 & 2.63 & 0.42 \\
\hline BDI-FS $^{\mid}$ & Online & 623 & 1.19 & 1.31 \\
\hline SPIN $^{\mathrm{a}}$ & Online & 623 & 2.33 & 1.67 \\
\hline MAQ ambivalent-worry $^{\mathrm{a}}$ & Online & 623 & 1.67 & 1.33 \\
\hline MAQ ambivalent-merger $^{\mathrm{a}}$ & \multicolumn{5}{|l}{} \\
\hline
\end{tabular}

Table 1. Means (or medians) and standard deviations (or interquartile ranges) of measured constructs. ${ }^{a}$ Normality can not be assumed, median and interquartile range are given. ${ }^{b}$ Normality can be assumed, mean and standard deviation are given.

Second item reduction. Genetic algorithms have increasingly been used for item selection in recent years ${ }^{26}$. Combining elements from random and guided search and allowing for the optimization of multiple, userdefined psychometric criteria regarding the entire item subset, meta-heuristics are powerful algorithms for complex, noisy search spaces ${ }^{27}$. A genetic algorithm was applied to the remaining item pool to optimize the model fit, reliability and discrimination between patients and healthy participants. The best item selection was found in six out of ten replications of the algorithm (see Supplementary Table S1 for item numbers and Supplement S2 for ready to use questionnaire versions in English and German). While the $\chi^{2}$-value was significant $\left(\chi^{2}=\right.$ $359.06, \mathrm{p}<0.001$ ), the descriptive fit measures now showed an acceptable to good model fit (RMSEA $=0.069$, $\mathrm{SRMR}=0.029, \mathrm{CFI}=0.970)$. The composite reliability was excellent $(0.940)$.

Substudy 3: factorial structure and content validity of the final scale. We cross-validated the factorial structure of the final item selection in a different part of the sample. For convergent and divergent validity, we then examined correlations with related constructs. We calculated group differences between patients and healthy participants, and different diagnostic groups to assess discriminant validity.

Measurement invariance and factorial structure. Metric invariance between the normal population and patient sample was supported $\left(C F I_{\Delta}=0.001\right)$, but not scalar invariance $\left(C F I_{\Delta}=0.005\right)$. Therefore, it can be assumed that the latent constructs have the same content meaning in the subpopulations studied, but groups should be compared with caution. Based on this model, the $\chi^{2}$-value was significant $\left(\chi^{2}=335.91, p<0.001\right)$. The RMSEA $=0.076$, the CFI $=0.970$ and the SRMR $=0.027$ indicated an acceptable to good fit. Standardized factor loadings ranged from $\lambda=0.561$ to $\lambda=0.856$. Detailed results can be found in Supplement S3.

Construct validity. Means and standard deviations of all measures can be found in Table 1. For the representative sample, these values can be understood as norm values. However, in the case of the clinical sample, which was not drawn representatively, they can only be understood as preliminary norms. We tested for mean differences between female and male participants. In the clinical sample we found no mean difference (Female: $M=2.30$, Male: $M=2.17, t=-1.14, p=0.259)$. In the representative sample, there was a significant gender difference found in the Wilcoxon ran sum test (Female: $M=1.90$, Male: $M=1.68, W=691,788, p<0.001$ ).

Consistent with our expectations, high correlations $(r>0.50)$ were observed with the IPSM, the SPIN, and the ambivalent-worry attachment subscale, closely with the BSI Interpersonal Sensitivity subscale (see Table 2). Furthermore, correlations with measures of depression, general psychopathological symptoms and the BSI Paranoid Ideation subscale were moderate. The correlation of the SPQ with the BDI-FS was unexpectedly high. The measures categorized as divergent showed small correlations, except for the unexpected moderate correlation of the SPQ with the BSI Phobic Anxiety subscale.

The construct validity was further supported by the correlation with the 18 -item version $(r=0.984)$, showing that the construct is adequately represented in the short version. The 10 -item version explained $97.7 \%$ of the variance of the 18 -item version.

Discriminant validity. We found significant differences between the clinical and the representative sample, as well as between patients with high and low rejection-sensitivity diagnoses (Table 3). 


\begin{tabular}{|c|c|c|c|c|}
\hline & Scale & $\mathbf{r}$ & Sample & Expectation supported $^{\mathrm{a}}$ \\
\hline \multicolumn{5}{|c|}{ Expected convergency } \\
\hline \multirow{4}{*}{ High } & IPSM & $0.69^{* *}$ & Clinical & $\sqrt{ }$ \\
\hline & BSI-interpersonal sensitivity & $0.47^{* *}$ & Clinical & $(\sqrt{ })$ \\
\hline & SPIN & $0.68^{* *}$ & Online & $\sqrt{ }$ \\
\hline & MAQ ambivalent-worry & $0.50^{* *}$ & Online & $\sqrt{ }$ \\
\hline \multirow{6}{*}{ Moderate } & BDI-FS & $0.64^{* *}$ & Online & $(\sqrt{ })$ \\
\hline & MAQ ambivalent-merger & $0.31^{* *}$ & Online & $\sqrt{ }$ \\
\hline & BSI-depression & $0.30^{* *}$ & Clinical & $\sqrt{ }$ \\
\hline & PHQ-4 & $0.24^{* *}$ & Representative & \\
\hline & BSI-global severity index & $0.34^{* *}$ & Clinical & $\sqrt{ }$ \\
\hline & BSI-paranoid ideation & $0.37^{* *}$ & Clinical & $\sqrt{ }$ \\
\hline \multicolumn{5}{|c|}{ Expected divergency } \\
\hline & MAQ secure & -0.06 & Online & $\sqrt{ }$ \\
\hline & GBB & $0.18^{\star *}$ & Representative & $\sqrt{ }$ \\
\hline & BSI-somatization & 0.14 & Clinical & $\sqrt{ }$ \\
\hline & BSI-phobic anxiety & $0.23^{* *}$ & Clinical & \\
\hline
\end{tabular}

Table 2. Correlations of the 10-item SPQ with convergent and divergent measures. Spearman rank correlations are used because normality could not be assumed for most measures. IPSM interpersonal sensitivity measure, $B S I$ brief symptom inventory, MAQ measure of attachment qualities, SPIN social phobia inventory, BDI-FS becks depression inventory - fast screen, $P H Q-4$ patient health questionnaire- $4 .{ }^{\text {a We }}$ expected highly convergent measures to show correlations $\geq 0.50$ and moderately convergent measures $\geq 0.30$. whereas divergent measures to show correlations $\leq 0.20 .{ }^{* *} \mathrm{p}<0.001 ;{ }^{\star} \mathrm{p}<0.05$.

\begin{tabular}{|l|l|l|l|l|l|}
\hline Sample/diagnosis & N & M & SD & t & p \\
\hline Representative sample $^{\mathrm{a}}$ & 2531 & 1.80 & 1.40 & & \\
\hline Clinical sample & 270 & 2.30 & 1.10 & -7.46 & $<0.001$ \\
\hline High rejection-sensitivity Disorders $^{\mathrm{b}}$ & 147 & 2.38 & 0.83 & & \\
\hline Low rejection-sensitivity disorders $^{\mathrm{c}}$ & 95 & 2.05 & 0.76 & -3.21 & 0.002 \\
\hline
\end{tabular}

Table 3. Comparison of SPQ values between representative and clinical sample and between patient subsamples with high or low rejection sensitivity. ${ }^{\text {a }} \mathrm{SP}$ for the representative sample is not normally distributed. Median and interquartile range are given, and Yuan-Bentler test is reported. ${ }^{b}$ Personality disorders, depression, social anxiety disorder. ${ }^{\mathrm{c}}$ Other anxiety disorders, trauma-related disorders.

A ROC analysis revealed poor discrimination between patients and healthy participants (AUC $=0.634$ ). The cut-off value of 1.83 showed a sensitivity of 0.678 and a specificity of 0.507 and classified $52.3 \%$ of participants accurately. The discriminatory power was higher for rejection-sensitive patients versus other participants $(\mathrm{AUC}=0.670)$. The determined cut-point $(\mathrm{SPQ}=2.16)$ classified $64.1 \%$ of the participants accurately (sensitivity $=0.640$; specificity $=0.641$ ).

\section{Discussion}

The aim of the studies was to develop and validate a brief self-report instrument assessing emotional reactions of individuals to social exclusion, rejection and relational devaluation. Based on the background of the socialphysical pain overlap theor $y^{4}$ and belongingness theory ${ }^{1}$, an initial pool was shortened in a two-step procedure, based on a total sample of 3424 respondents, to a final version with 10 items. We found that the final SPQ was associated with a solid one-factor-structure and excellent reliability.

To establish construct validity, we used rating scales of related constructs to demonstrate convergent validity. Most closely related to the target construct, the Interpersonal Sensitivity Measure ${ }^{22}$, which measures perception or anticipation of criticism or rejection by others, demonstrated the highest correlation. A high correlation was also found with the BSI-subscale Interpersonal Sensitivity, which assesses feelings of personal inadequacy and inferiority ${ }^{28}$, reflecting most evidently the convergent validity of the SPQ. Furthermore, as predicted by the interpersonal theory of Leary ${ }^{5}$, we also found significant, although moderate correlations to anxious-ambivalent attachment. Thus, individuals high on social pain might be characterized by a high need for intimacy on one side, and a high level of neuroticism and low level of openness to experience on the other side ${ }^{29}$.

In addition, given the close theoretical relationship to social pain, we expected moderate to high correlations with measures of depression ${ }^{11,13}$ and social anxiety ${ }^{16,30}$. These expectations were confirmed, in particular for depression scales which cover not only core symptoms of depression but also interpersonal problems. Interestingly, the high correlation with the BSI-subscale Paranoid Ideation indicates that high SPQ values may also 
reflect disordered thinking like projective thoughts and suspiciousness in transition to more severe psychiatric disorders, such as paranoid delusions.

We also tested the discriminant validity of the SPQ by comparing clinical subgroups with diagnoses related to varying degrees to social pain. In line with our expectations, patients with depression, SAD and personality disorders showed higher levels of social pain than patients with other diagnoses (anxiety disorders other than $\mathrm{SAD}$, trauma-related disorders). In addition, our clinical sample showed significantly elevated scores in SPQ compared to the representative sample. Interestingly, the scores were normally distributed in the clinical sample showed stronger skewness in the normal population (clinical sample $=-0.05$, representative sample $=0.09$ ). In the representative sample a considerable number of participants $(n=113)$ had a score of exactly 0 , which was not true for the clinical sample. This supports the subclinical nature of the construct. To identify patients with increased social pain for specific psychological interventions, we determined preliminary cut-off values based on the comparisons of the clinical sample with the representative sample, and of patients with rejection-sensitive diagnoses with clinical and non-clinical controls. However, the identification of patients with high sensitivity for rejection allowed for a higher rate of correct classification (64.1\%) than individuals with mental disorders $(52.3 \%)$. Thus, it seems to be more appropriate to use the SPQ cut-off to identify patients with high individual susceptibility to social pain, rather than for the identification of mental disorders.

The present study has several limitations.

First, we did not involve expert ratings on the relevance of the items in the development of the item pool, which would have increased content validity ${ }^{31}$. However, the questionnaire was developed in German and no experts were available for content ratings. Therefore, we strongly based the definition of items on theoretical considerations, deriving a predefined set of situational variables and emotional reactions reported from literature.

Second, for the demonstration of convergent validity, although we defined apriori criteria for convergency and used descriptive analyses of the size of correlations, data from other sources such as cyberball experiments may be more conclusive.

Third, we included samples which were recruited in different ways. The online survey was a convenience sample, and self-selection may have influenced the characteristics of the sample. Furthermore, the clinical sample was much smaller than the others, with limited options to create sufficiently large diagnostic subsamples. Thus, also the SPQ cut-off value we obtained here is only preliminary.

Fourth, the test-retest reliability of the SPQ was not examined, which needs to be addressed in future studies.

Fifth, due to the lack of latent measurement invariance, group comparisons should be interpreted with care. Future studies are needed to investigate the causes of the measurement invariance problems. For now, the scale seems to be more appropriate for comparisons between patients.

Finally, the data-driven selection approach poses the risk of over-fitting, but we mitigated this problem by reserving a portion of the sample for independent cross-validation.

In conclusion, apart from these limitations, the SPQ in this study was associated with robust psychometric properties with regard to factor structure, reliability, and convergent and discriminant validity, which supports its use in clinical and nonclinical populations and may close a gap in the assessment of social pain. It should be noted that the process of item selection favored items referring not to specific persons, such as family members or partners. However, since the SPQ is primarily designed to screen for social pain in clinical populations, therapists and counsellors should explore in the individual case more details about the triggers and persons associated with social pain. The next phase of research will include experimental studies to investigate the prediction of emotional reactions in cyberball experiments with clinical samples of depressed and socially anxious patients ${ }^{30}$. Furthermore, it will be interesting to tailor interventions such as cognitive restructuring, behavioral experiments, or acceptance and commitment therapy to the needs of patients with high social pain ${ }^{32}$, and to assess treatment sensitivity in future intervention trials.

\section{Methods and measures}

Sample. We recruited three samples during the study. All methods were carried out in accordance with relevant guidelines and regulations. Study 1 and Study 3 were approved by the research ethics board of the Department of Psychology, Goethe University Frankfurt, and Study 2 was approved the ethics board of the Department of Medicine, University of Leipzig. Informed consent was obtained from all subjects or, if subjects are under 18, from a parent and/or legal guardian. No compensation was given for participation.

The first sample was recruited via the internet, local newspapers, and flyers. The only exclusion criterion was a minimum age of 17 years. The sample comprised 623 individuals with a mean age of $32.61(S D=11.13)$. $39 \%$ of the participants reported a mental disorder, most frequently depression (22.8\%).

The second sample was recruited in a national, representative general population survey. The data were collected in 2 waves between May and June 2019 by professional demographic consultants (Unabhängige Serviceeinrichtung für Umfragen, Methoden und Analysen, Berlin). 2531 households were eligible to participate and were visited by trained face-to-face interviewers who recorded participants' demographic information; other information was collected through a paper/pencil self-report.

Data for the third sample were collected between June 2018 and October 2019. Patients who were admitted to psychotherapy at the Center for Cognitive Behavioral Therapy of the Goethe University Frankfurt were informed about the goals of the study. Inclusion criteria comprised at least one diagnosis of mental disorder according to ICD-10, and an age of 18 years or more. The participation was voluntary and not part of the treatment. Participants were invited to complete the questionnaires on PC before starting treatment. Sociodemographic characteristics for the online sample $(n=623)$, the representative sample $(n=2531)$ and the patient sample $(n=270)$ are given in Table 4 . 


\begin{tabular}{|c|c|c|c|}
\hline & $\begin{array}{l}\text { Online } \\
\mathrm{N}=623\end{array}$ & $\begin{array}{l}\text { Representative } \\
\mathrm{N}=2531\end{array}$ & $\begin{array}{l}\text { Clinical } \\
\mathrm{N}=270\end{array}$ \\
\hline \multicolumn{4}{|l|}{ Age (years) } \\
\hline $\mathrm{M}(\mathrm{SD})$ & $32.61(11.13)$ & $48.44(17.86)$ & $37.23(12.79)$ \\
\hline Range & $17-66$ & $17-95$ & $19-74$ \\
\hline \multicolumn{4}{|l|}{ Gender } \\
\hline Female & & $64,0 \%$ & $62.6 \%$ \\
\hline Male & & $46.0 \%$ & $37.4 \%$ \\
\hline \multicolumn{4}{|l|}{ Marital status } \\
\hline Single & $41.40 \%$ & $30.0 \%$ & $42.5 \%$ \\
\hline Firm partnership & $31.30 \%$ & $31.30 \%$ & $20.4 \%$ \\
\hline Married & $19.90 \%$ & $43.5 \%$ & $26.6 \%$ \\
\hline Divorced & $7.40 \%$ & $14.5 \%$ & $8.7 \%$ \\
\hline \multicolumn{4}{|l|}{ Education } \\
\hline No degree & $1.80 \%$ & $2.4 \%$ & $3.7 \%$ \\
\hline Elementary school & $7.40 \%$ & $27.2 \%$ & $11.2 \%$ \\
\hline High school diploma & $19.30 \%$ & $39.8 \%$ & $18.5 \%$ \\
\hline Bachelor degree & $35.30 \%$ & $17.4 \%$ & $34.1 \%$ \\
\hline Master degree & $36.30 \%$ & $9.9 \%$ & $25.9 \%$ \\
\hline Not specified & & $3.3 \%$ & $6.6 \%$ \\
\hline \multicolumn{4}{|l|}{ Employment } \\
\hline Employed & $45.60 \%$ & $59.3 \%$ & $73.7 \%$ \\
\hline Unemployed & $7.90 \%$ & $4.1 \%$ & $3.0 \%$ \\
\hline Homemaker & $3.50 \%$ & $3.4 \%$ & $2.6 \%$ \\
\hline Student/school & $32.90 \%$ & $6.8 \%$ & $13.4 \%$ \\
\hline In training & $4.30 \%$ & $1.7 \%$ & $2.1 \%$ \\
\hline Retired & $5.80 \%$ & $24.5 \%$ & $5.1 \%$ \\
\hline \multicolumn{4}{|l|}{ Primary diagnoses } \\
\hline Depression & & & $31.1 \%$ \\
\hline Social anxiety disorder & & & $14.9 \%$ \\
\hline Other anxiety disorders & & & $17.3 \%$ \\
\hline Trauma-related disorders & & & $19.6 \%$ \\
\hline Personality disorder & & & $10.0 \%$ \\
\hline Other diagnoses & & & $6.3 \%$ \\
\hline
\end{tabular}

Table 4. Sociodemographic characteristics of the three samples.

Statistical analysis. Normality for all measures was tested using the Shapiro-Wilk test and inspection of Q-Q plots. The SPQ-10 test scores were normally distributed in the patient sample $(W=0.99, p=0.14)$, but not in the representative sample $(W=0.98, p<0.001)$. Normal distribution could not be assumed for several of the measures, especially clinical and subclinical measures, which is consistent with theory. Therefore, we use nonparametric methods where necessary, e.g. Spearman rank correlation, robust linear regression etc.

The factorial structure and model fit of the questionnaire were checked at several stages by means of confirmatory factor analysis (CFA), using the R-package lavaan ${ }^{33}$. Since normality could not be assumed in all cases, we used the robust maximum likelihood estimator (MLR), that provides more accurate results than maximum likelihood estimation ${ }^{34}$. In addition to the $\chi^{2}$-test, we used the root mean square error of approximation (RMSEA) and the standardized root mean square residual (SRMR) as assessment for the absolute fit and the comparative fit index (CFI) for the relative fit. RMSEA and SRMR values of $<0.05$ and $<0.08$; and a CFI of $>0.97$ and $>0.95$ are considered as good and acceptable, respectively ${ }^{35}$.

For the first item reduction, items with a loading of $\lambda>0.60$ were selected. The final selection was carried out using a genetic algorithm (GA) ${ }^{36}$ implemented in the R-package $s_{\text {tuart }}{ }^{37}$. Within the stuart-framework, all parameters derived from CFA can be included in the objective function. In this study, we optimized RMSEA and SRMR, model-based reliability and discrimination between patients and healthy participants. Since metaheuristics solve optimization problems probabilistically, the algorithm was applied ten times to ensure reliability. Additional details regarding the application of the genetic algorithm can be found online in Supplement S4. The full sample was randomly split into a construction $(n=1401)$ and a validation sample $(n=1400)$ for unbiased cross-validation.

Measurement invariance between the representative and the clinical sample was evaluated using a stepwise procedure $^{38}$ with a cut-off value of $\Delta \mathrm{CFI} \geq 0.005^{39}$. 
To assess convergent and divergent validity, Spearman rank correlations with measures of convergent (interpersonal sensitivity, attachment styles, depression and social anxiety) and divergent (somatic complaints) dimensions were calculated from the three samples.

To assess discriminant validity, patients with primary diagnoses of personality disorders, depression and social anxiety disorders $(n=147)$, empirically related to rejection sensitivity or social pain, were compared to patients with other anxiety disorders or trauma-related disorders $(n=95)$. The Yuan-Bentler test was used for comparisons where normality could not be assumed. A ROC analysis was conducted to determine the discriminatory power for the discrimination of patients from healthy participants, and of patients with rejection-sensitive diagnoses from all other participants. A cut-off point was calculated by maximizing the sum of sensitivity and specificity.

All statistical tests were conducted with $\alpha=0.05$. Analyses were conducted with the R statistical environment, using the packages robustbase ${ }^{40}, p$ Roc $^{41}$ and cutpointr ${ }^{42}$.

Measures. The Social Phobia Inventory (SPIN) $)^{43}$ is a 17 -item screening instrument which evaluates fear, avoidance, and physiological discomfort in a variety of social situations. The internal consistency (Cronbach's $\alpha=0.82-0.95$, here $\omega=0.94$ ) and convergent and divergent validity ${ }^{43-45}$.

The Measure of Attachment Qualities (MAQ) ${ }^{46}$ is a 14 -item self-report measure of adult attachment patterns. It comprises subscales of secure and avoidant attachment, ambivalent-worrying and ambivalent/merged. Internal consistency was moderate (Cronbach's $\alpha=0.70$ ). Individuals high in anxious attachment tend to be sensitive to rejection cues ${ }^{47}$.

The Patient Health Questionnaire-4 (PHQ-4) ${ }^{48}$ is a brief screening scale for anxiety and depression. Four items refer to common symptoms of anxiety and depression. The PHQ-4 has been validated in clinical ${ }^{48}$ and general population ${ }^{49}$ samples.

The Somatic Complaints Inventory $(\mathrm{GBB})^{50}$ was used to assess physical well-being. Eight items refer to somatic complaints. In large epidemiological samples, reliability, item characteristics, and factor structure of the GBB were excellent ${ }^{51}$, here internal consistency was high $(\omega=0.89)$.

The Brief Symptom Inventory (BSI) ${ }^{28}$ is a 53 -item-self-report instrument to assess psychological symptoms. It is composed of nine primary symptom dimensions. The total score (Global Severity Index) measures the overall psychological distress. In psychiatric patients, internal consistencies $(\alpha=0.71-0.85$; present study: $\omega=0.73$ to $0.85)$ and test-retest reliabilities $\left(r_{t t}=0.68-0.91\right)$ of the subscales are high. The BSI has shown a high convergent, discriminant, and construct validity in clinical samples ${ }^{52}$.

The Interpersonal Sensitivity Measure (IPSM) ${ }^{22}$ is a 36-item self-report instrument assessing perception or anticipation of criticism and rejection. The instrument proved as reliable (here $\omega=0.91$ ) and valid in predicting the development of depressive episodes and low self-esteem ${ }^{53}$, social anxiety disorder ${ }^{24}$, and persecutory ideations ${ }^{54}$.

Beck depression inventory-fast screen (BDI-FS; Beck et al. ${ }^{55}$ ). The BDI-FS is a seven-item self-report inventory designed to evaluate depression in patients with medical illness. It has been proven a reliable and valid measure of depression in the German general population ${ }^{56}$, with an internal consistency of $\alpha=0.84$.

\section{Data availability}

The datasets generated during and/or analysed during the current study are available in the OSF repository (https://osf.io/m78sx/?).

\section{Code availability}

The full code of the genetic algorithm application is available in the OSF repository (https://osf.io/m78sx/?).

Received: 17 November 2020; Accepted: 26 March 2021

Published online: 15 April 2021

\section{References}

1. Baumeister, R. F. \& Leary, M. R. The need to belong: Desire for interpersonal attachments as a fundamental human motivation. Psychol. Bull. 117, 497-529 (1995).

2. Vangelisti, A. L. \& Brody, N. The physiology of social pain: Examining, problematizing, and contextualizing the experience of social pain. In The Oxford Handbook of the Physiology of Interpersonal Communication (eds Aloia, L. S. et al.) 47-68 (Oxford University Press, 2020).

3. Leary, M. R. \& Springer, C. A. Hurt feelings: The neglected emotion. In Behaving Badly: Aversive Behaviors in Interpersonal Relationships (ed. Kowalski, R. M.) 151-175 (American Psychological Association, 2001).

4. Eisenberger, N. I., Lieberman, M. D. \& Williams, K. D. Does rejection hurt? An FMRI study of social exclusion. Science 302, 290-292 (2003).

5. Leary, M. R., Haupt, A. L., Strausser, K. S. \& Chokel, J. T. Calibrating the sociometer: The relationship between interpersonal appraisals and state self-esteem. J. Pers. Soc. Psychol. 74, 1290-1299 (1998).

6. Leary, M. R. \& Baumeister, R. F. The nature and function of self-esteem: Sociometer theory. In Advances in Experimental Social Psychology Vol. 32 (eds Leary, M. R. \& Baumeister, R. F.) 1-62 (Academic Press, 2000).

7. Mwilambwe-Tshiloboa, L. \& Spreng, R. N. Social exclusion reliably engages the default network: A meta-analysis of Cyberball. Neuroimage 227, 117666. https://doi.org/10.1016/j.neuroimage.2020.117666 (2020).

8. Hartgerink, C. H. J., van Beest, I., Wicherts, J. M. \& Williams, K. D. The ordinal effects of ostracism: A meta-analysis of 120 cyberball studies. PLoS ONE 10, e0127002. https://doi.org/10.1371/journal.pone.0127002 (2015).

9. American Psychiatric Association. Diagnostic and Statistical Manual of Mental Disorders (American Psychiatric Association, 2013).

10. McLaughlin, K. A., Colich, N. L., Rodman, A. M. \& Weissman, D. G. Mechanisms linking childhood trauma exposure and psychopathology: A transdiagnostic model of risk and resilience. BMC Med. https://doi.org/10.1186/s12916-020-01561-6 (2020). 
11. Gao, S., Assink, M., Cipriani, A. \& Lin, K. Associations between rejection sensitivity and mental health outcomes: A meta-analytic review. Clin. Psychol. Rev. 57, 59-74 (2017).

12. Poggi, A., Richetin, J. \& Preti, E. Trust and rejection sensitivity in personality disorders. Curr. Psychiatry Rep. https://doi.org/10. 1007/s11920-019-1059-3 (2019).

13. Reinhard, M. A. et al. The vicious circle of social exclusion and psychopathology: A systematic review of experimental ostracism research in psychiatric disorders. Eur. Arch. Psychiatry Clin. Neurosci. 270, 521-532 (2020).

14. Jobst, A. et al. Effects of social exclusion on emotions and oxytocin and cortisol levels in patients with chronic depression. $J$. Psychiatr. Res. 60, 170-177 (2015).

15. Seidl, E. et al. Response to ostracism in patients with chronic depression, episodic depression and borderline personality disorder a study using Cyberball. J. Affect. Disord. 260, 254-262 (2020).

16. Fung, K. \& Alden, L. E. Once hurt, twice shy: Social pain contributes to social anxiety. Emot. Wash. DC 17, 231-239 (2017)

17. Nietlisbach, G. \& Maercker, A. Social cognition and interpersonal impairments in trauma survivors with PTSD. J. Aggress. Maltreat. Trauma 18, 382-402 (2009).

18. Zadro, L., Williams, K. D. \& Richardson, R. How low can you go? Ostracism by a computer is sufficient to lower self-reported levels of belonging, control, self-esteem, and meaningful existence. J. Exp. Soc. Psychol. 40, 560-567 (2004).

19. Besikci, E., Dvir, M. \& Williams, K. Ostracism. In Encyclopedia of Personality and Individual Differences (eds Zeigler-Hill, V. \& Shackelford, T. K.) 1-6 (Springer, 2017).

20. Gerber, J. P., Chang, S.-H. \& Reimel, H. Construct validity of Williams' ostracism needs threat scale. Personal. Individ. Differ. 115, 50-53 (2017).

21. Berenson, K. R. et al. Rejection sensitivity and disruption of attention by social threat cues. J. Res. Personal. 43, 1064-1072 (2009).

22. Boyce, P. \& Parker, G. Development of a scale to measure interpersonal sensitivity. Aust. N. Z. J. Psychiatry 23, 341-351 (1989).

23. Innamorati, M. et al. Construct validity and reliability of the adult rejection sensitivity questionnaire: A comparison of three factor models. Depress. Res. Treat. 2014, 1-10 (2014).

24. Harb, G. C., Heimberg, R. G., Fresco, D. M., Schneier, F. R. \& Liebowitz, M. R. The psychometric properties of the interpersonal sensitivity measure in social anxiety disorder. Behav. Res. Ther. 40, 961-979 (2002).

25. Leary, M. R., Springer, C., Negel, L., Ansell, E. \& Evans, K. The causes, phenomenology, and consequences of hurt feelings. J. Pers. Soc. Psychol. 74, 1225-1237 (1998).

26. Basarkod, G., Sahdra, B. \& Ciarrochi, J. Body image-acceptance and action questionnaire-5: An abbreviation using genetic algorithms. Behav. Ther. 49, 388-402 (2018).

27. Boussaïd, I., Lepagnot, J. \& Siarry, P. A survey on optimization metaheuristics. Inf. Sci. 237, 82-117 (2013).

28. Derogatis, L. R. BSI Brief Symptom Inventory (Pearson, 1993).

29. Shaver, P. R. \& Brennan, K. A. Attachment styles and the 'Big Five' personality traits: Their connections with each other and with romantic relationship outcomes. Pers. Soc. Psychol. Bull. 18, 536-545 (1992).

30. Hudd, T. \& Moscovitch, D. A. Coping with social wounds: How social pain and social anxiety influence access to social rewards. J. Behav. Ther. Exp. Psychiatry 68, 101572 (2020).

31. Worthington, R. L. \& Whittaker, T. A. Scale development research: A content analysis and recommendations for best practices. Couns. Psychol. 34, 806-838 (2006).

32. Ducasse, D. et al. Acceptance and commitment therapy for the management of suicidal patients: A randomized controlled trial. Psychother. Psychosom. 87, 211-222 (2018).

33. Rosseel, Y. lavaan: An R package for structural equation modeling. J. Stat. Softw. 48, 1-36 (2012).

34. Gao, C., Shi, D. \& Maydeu-Olivares, A. Estimating the maximum likelihood root mean square error of approximation (RMSEA) with non-normal data: A Monte-Carlo study. Struct. Equ. Model. Multidiscip. J. 27, 192-201 (2020).

35. Schermelleh-Engel, K., Moosbrugger, H. \& Müller, H. Evaluating the fit of structural equation models: tests of significance and descriptive goodness-of-fit measures. Methods Psychol. Res. Online 8, 23-74 (2003).

36. Schroeders, U., Wilhelm, O. \& Olaru, G. Meta-heuristics in short scale construction: Ant colony optimization and genetic algorithm. PLoS ONE 11, e0167110 (2016).

37. Schultze, M. stuart: Subtests Using Algorithmic Rummaging Techniques (2020).

38. Putnick, D. L. \& Bornstein, M. H. Measurement invariance conventions and reporting: The state of the art and future directions for psychological research. Dev. Rev. 41, 71-90 (2016).

39. Chen, F. F. Sensitivity of goodness of fit indexes to lack of measurement invariance. Struct. Equ. Model. Multidiscip. J. 14, 464-504 (2007).

40. Maechler, M. et al. Robustbase: Basic Robust Statistics R Package (2020).

41. Robin, X. et al. pROC: An open-source package for R and S+ to analyze and compare ROC curves. BMC Bioinform. https://doi. org/10.1186/1471-2105-12-77 (2011).

42. Thiele, C. \& Hirschfeld, G. cutpointr: Improved Estimation and Validation of Optimal Cutpoints in R (2020).

43. Connor, K. M. et al. Psychometric properties of the social phobia inventory (SPIN): New self-rating scale. Br. J. Psychiatry 176, 379-386 (2000).

44. de Osório, F. L., Crippa, J. A. S. \& Loureiro, S. R. Further study of the psychometric qualities of a brief screening tool for social phobia (MINI-SPIN) applied to clinical and nonclinical samples. Perspect. Psychiatr. Care 46, 266-278 (2010).

45. Sosic, Z., Gieler, U. \& Stangier, U. Screening for social phobia in medical in- and outpatients with the German version of the Social Phobia Inventory (SPIN). J. Anxiety Disord. 22, 849-859 (2008).

46. Carver, C. S. Adult attachment and personality: Converging evidence and a new measure. Pers. Soc. Psychol. Bull. 23, 865-883 (1997).

47. Mikulincer, M., Shaver, P. R. \& Pereg, D. Attachment theory and affect regulation: The dynamics, development, and cognitive consequences of attachment-related strategies. Motiv. Emot. 27, 77-102 (2003).

48. Kroenke, K., Spitzer, R. L., Williams, J. B. W. \& Löwe, B. An ultra-brief screening scale for anxiety and depression: The PHQ-4. Psychosomatics 50, 613-621 (2009).

49. Löwe, B. et al. A 4-item measure of depression and anxiety: Validation and standardization of the Patient Health Questionnaire-4 (PHQ-4) in the general population. J. Affect. Disord. 122, 86-95 (2010).

50. Brähler, E. \& Der Scheer, J. W. Gießener Beschwerdebogen (GBB) (The Giessen Somatic Complaint Scale). Handbuch (Manual) (Huber, 1995).

51. Kliem, S. et al. Brief assessment of subjective health complaints: Development, validation and population norms of a brief form of the Giessen Subjective Complaints List (GBB-8). J. Psychosom. Res. 95, 33-43 (2017).

52. Geisheim, C. et al. Das brief symptom inventory (BSI) als Instrument zur Qualitätssicherung in der Psychotherapie (The German version of the brief symptom inventory (BSI): Reliability and validity in a sample of outpatient psychotherapy patients). Diagnostica 48, 28-36 (2002).

53. Boyce, P., Parker, G., Barnett, B., Cooney, M. \& Smith, F. Personality as a vulnerability factor to depression. Br. J. Psychiatry 159, 106-114 (1991).

54. Masillo, A. et al. Interpersonal sensitivity in the at-risk mental state for psychosis. Psychol. Med. 42, 1835-1845 (2012).

55. Beck, A.T. et al. BDI-Fast Screen for Medical Patients: Manual. (Psychological Corporation, 2000). 
56. Kliem, S., Mößle, T., Zenger, M. \& Brähler, E. Reliability and validity of the beck depression inventory-fast screen for medical patients in the general German population. J. Affect. Disord. 156, 236-239 (2014).

\section{Author contributions}

U.S. contributed to the study conception and design. Material preparation and data collection were performed by U.S. and E.B. The first draft of the manuscript was written by U.S. and J.S. and all authors commented on previous versions of the manuscript. All authors read and approved the final manuscript.

\section{Funding}

Open Access funding enabled and organized by Projekt DEAL.

\section{Competing interests}

The authors declare no competing interests.

\section{Additional information}

Supplementary Information The online version contains supplementary material available at https://doi.org/ 10.1038/s41598-021-87351-3.

Correspondence and requests for materials should be addressed to U.S.

Reprints and permissions information is available at www.nature.com/reprints.

Publisher's note Springer Nature remains neutral with regard to jurisdictional claims in published maps and institutional affiliations.

(c) (i) Open Access This article is licensed under a Creative Commons Attribution 4.0 International License, which permits use, sharing, adaptation, distribution and reproduction in any medium or format, as long as you give appropriate credit to the original author(s) and the source, provide a link to the Creative Commons licence, and indicate if changes were made. The images or other third party material in this article are included in the article's Creative Commons licence, unless indicated otherwise in a credit line to the material. If material is not included in the article's Creative Commons licence and your intended use is not permitted by statutory regulation or exceeds the permitted use, you will need to obtain permission directly from the copyright holder. To view a copy of this licence, visit http://creativecommons.org/licenses/by/4.0/.

(C) The Author(s) 2021 